\title{
INFRARED LIGHT CURVES OF CARBON-RICH VARIABLES
}

\author{
PATRICIA WHITELOCK \\ South African Astronomical Observatory \\ Observatory 7935, South Africa
}

\begin{abstract}
Long-term trends in the infrared ( $J H K L)$ light curves of various carbon variables are described. Some stars, e.g. the semi-regular variables R Scl and GM CMa, show multiple periodicities; others, particularly the Miras with moderately thick dust-shells, show more erratic long-term changes. The light curves for $\mathrm{R}$ For, which have been intensively monitored over 20 years, show a pattern which is reminiscent of that seen for $\mathrm{R} \mathrm{CrB}$ stars. This pattern is superimposed on regular large-amplitude Mira pulsations. The multi-periodic and erratic behaviour of these stars is compared with the predictions from various models.
\end{abstract}

\section{Introduction}

Intrinsic carbon stars are the consequence of dredge-up during the helium shell-flash cycle near the top of the Asymptotic Giant Branch (AGB). It is well established that copious mass loss occurs in low and intermediate mass stars towards the end of their AGB evolution. It is also apparent that pulsating stars have particularly high mass-loss rates, strongly suggesting a causal relationship between mass loss and pulsation. It is, however, unclear how either mass loss or pulsation is affected by the changes in stellar structure which accompany the helium shell-flash cycles.

This paper describes some results from a long-term programme in progress at the South African Astronomical Observatory (SAAO) to monitor the infrared magnitudes of carbon stars. The programme is intended to investigate the relationship between pulsation and mass loss over many pulsation cycles and to look for long-term trends. A number of people have been involved in this programme over the years, most notably Michael Feast who initiated the project over 20 years ago. The observations are made through broad-band $J H K L$ filters using the MKII infrared photometer 
on the $0.75-\mathrm{m}$ telescope at Sutherland in South Africa. It is particularly appropriate and useful to work in the 1 to $4 \mu \mathrm{m}$ region when dealing with carbon variables. First, because they are cool stars and the stellar energy distributions usually peak around $1 \mu \mathrm{m}$, we are examining the behaviour of the bolometric output rather than minor fluctuations in temperature. Secondly, most of these stars have significant dust shells, and the light curve in $L(3.45 \mu \mathrm{m})$ is principally sampling this dust shell.

One of the principal interests in this work was in studying the properties of the Mira variables, both carbon- and oxygen-rich. Miras are understood to be the last reasonably long-lived phase in the evolution of low and intermediate mass stars. They are at the top of the AGB just prior to the journey across the HR diagram to become white dwarfs. The most informative thing about a Mira is its pulsation period. For oxygen-rich Miras there are various correlations that suggest the pulsation period of the Mira tells us about the population from which it has evolved. In particular the kinematics and scale heights of Miras in the solar neighbourhood tell us that the short-period stars belong to a thick-disk population while the longer period stars are kinematically cooler (Whitelock 1995 and references therein). The Miras obey a period-luminosity relation. Feast et al. (1989) showed that oxygen- and carbon-rich Miras in the LMC obey the same PL relation in $K(2.2 \mu \mathrm{m})$. More recently, Groenewegen \& Whitelock (1996) established that they also obey the same bolometric period-luminosity relation.

\section{Infrared Colours}

It is instructive to examine the colours of carbon-rich variables in comparison to those of oxygen-rich stars. Figure 1 shows the colours for carbon variables; they have been corrected for reddening using a galactic model of the extinction. These are mean colours; the individual observations often show a good deal of spread. In particular, the colours of stars which undergo obscuration events (see below) are much redder during the faint phases than otherwise. The illustrated stars have not been selected in any systematic way. They include bright local variables which are part of our long-term monitoring program, as discussed below. They also include some carbon-rich stars with infrared excesses that were selected on the basis of their galactic coordinates as potential probes of galactic structure. Some have been very well studied, others much less so. Note that it is not always easy to distinguish between Miras and other types of variable on the basis of limited infrared observations.

Figure 1 shows a complete mixture of Mira and other variables; there is no easy way to separate them on the basis of these colours. This is in marked contrast to similar diagrams for oxygen-rich stars, e.g. Fig. 3 of Whitelock 


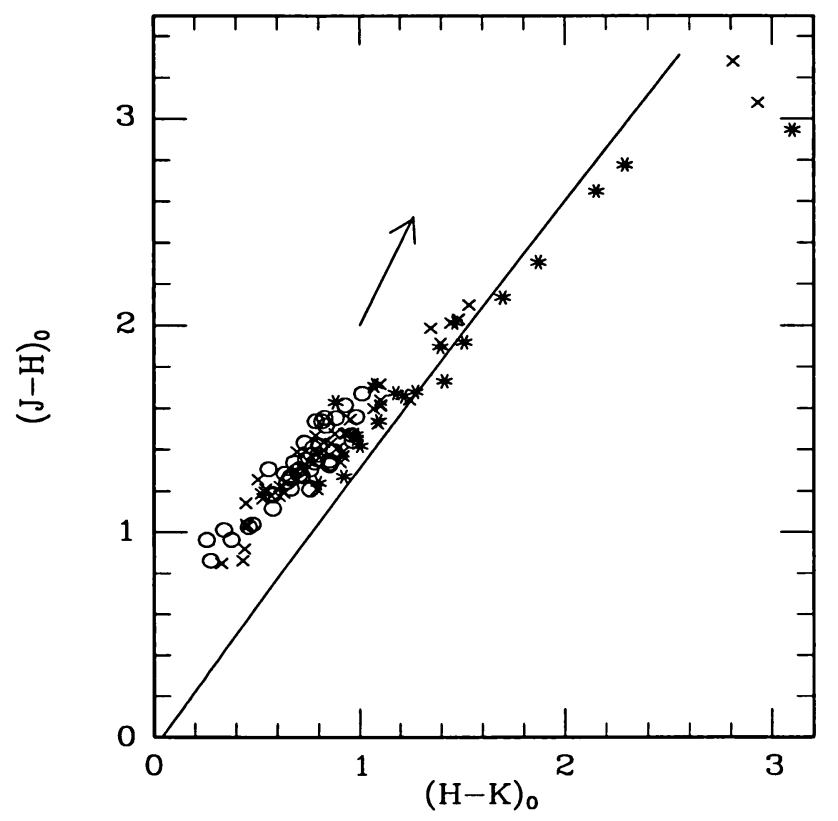

Figure 1. The mean infrared colours of carbon variables. The stars discussed below have the following $(H-K)_{0},(J-H)_{0}$ colours: R For $(1.27,1.68)$; TT Cen $(0.51,1.26)$; IZ Peg (3.10, 2.95); R Scl $(0.77,1.38)$; GM CMa $(0.89,1.41)$. Crosses are Miras, open circles are semi-regular variables, and asterisks are Miras with large-amplitude long-period or erratic changes. The line is the locus of blackbodies of various temperatures and the arrow represents a reddening vector of $A_{V}=5 \mathrm{mag}$.

et al. (1994), where the Miras are clearly separated from the other stars. It is the strong $\mathrm{H}_{2} \mathrm{O}$ features in the very extended atmospheres of these O-rich Miras that are the major influence on their colours. The only clear trend among the carbon stars in Fig. 1 is that the stars with the reddest colours, those with the thickest dust shells, are Miras and not small-amplitude variables, and that there is a large fraction of large-amplitude erratic variables among these stars with large excesses. This is of course expected if the erratic and long-term variations are caused by dust obscuration.

\section{R Fornacis}

R Fornacis is a bright, well-studied carbon Mira. Its $J$ light curve is shown in Figure 2. This comprises 153 observations taken over more than 20 years. The early half of this light curve has been discussed by Feast et al. (1984) and by Le Bertre (1988). Both papers conclude that the faint phase in 1983, around JD 2445600, was caused by increased dust obscuration. The combined SAAO and ESO data from JD 2444900 to JD 2447600 were discussed by Winters et al. (1994). They suggest that such dust-obscuration events 


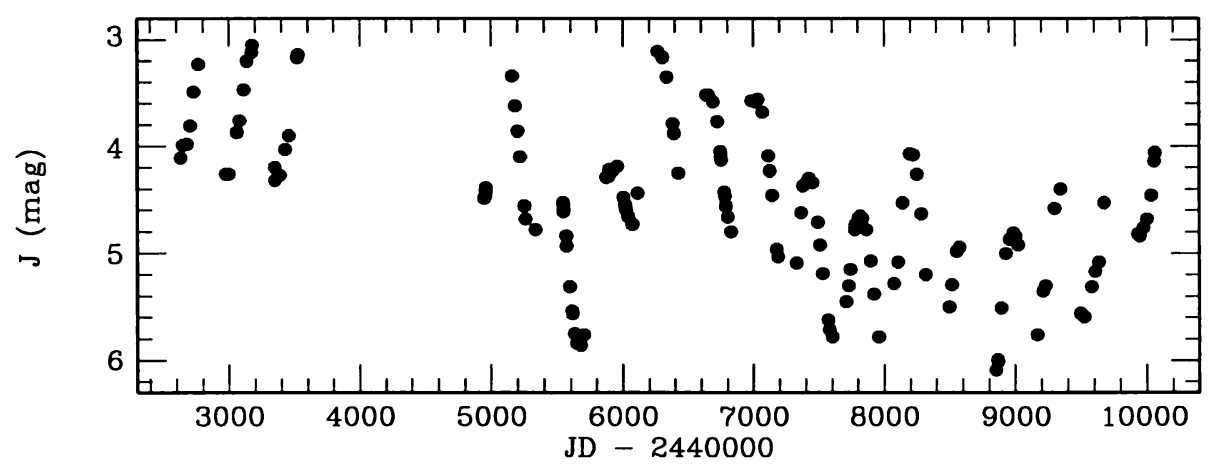

Figure 2. The $J(1.25 \mu \mathrm{m})$ light curve of $\mathrm{R}$ For.

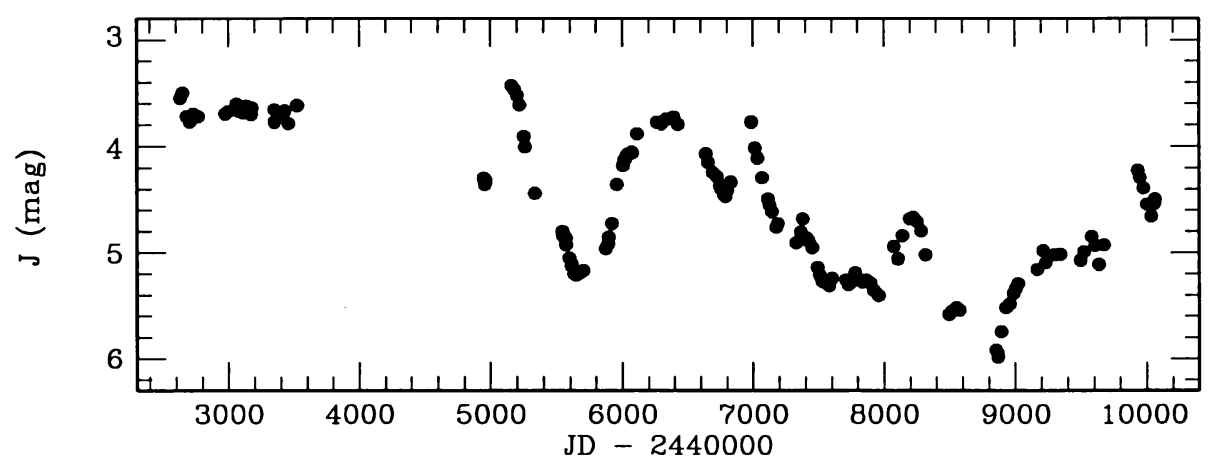

Figure 3. The $J(1.25 \mu \mathrm{m})$ light curve of $\mathrm{R}$ For after removing pulsational variations.

could occur periodically.

The GCVS gives the pulsation period of $\mathrm{R}$ For as 388.7 days. A Fourier analysis of the $J$ light curve gives $386.6 \pm 1.3 \mathrm{~d}$ for the complete data-set, which is not significantly different from the GCVS value. If the light curve is divided up, then a slightly different period is determined for each of the different time slots: $379.9 \pm 3$ for JD $2442630-3525,403.1 \pm 6$ for JD $2444947-$ 6828 , and $381.4 \pm 3$ for JD 2446986-50062. The early and later data show essentially the same value while the period in between is longer, and the amplitude larger. It is difficult to say how meaningful these differences are and their adoption does not affect the conclusions of this study.

Figure 3 shows the $J$ light curve with the pulsation removed assuming the periods listed above for the different time slots. Although there are a few places where the pulsation appears to have been imperfectly removed (e.g. around JD 2447000), the overall impression is that of a smooth variation of the mean magnitude once the pulsational variations have been removed.

The $H$ and $K$ data are similar to $J$ and are not illustrated here. Figure 4 


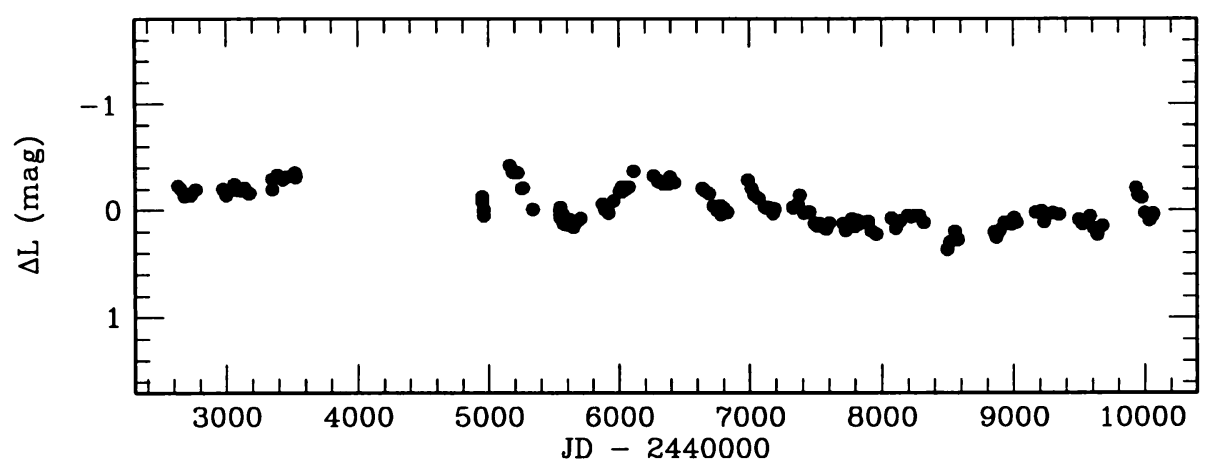

Figure 4. The $L(3.45 \mu \mathrm{m})$ light curve of $\mathrm{R}$ For after removing pulsational variations.

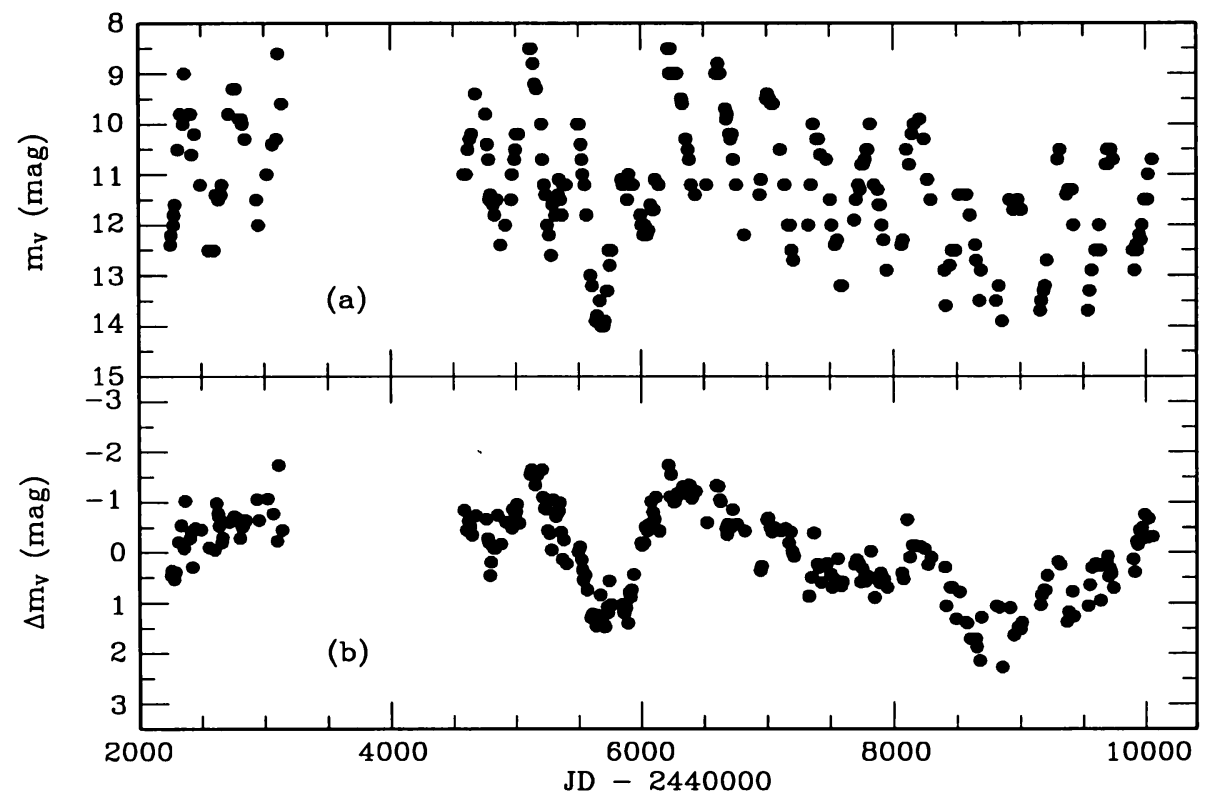

Figure 5. (a) Visual light curve for R For from observations by Danie Overbeek. (b) The same after removing pulsational variations.

shows the $L$ data, on the same scale as Fig. 3, after removal of the pulsation in exactly the same way as for the $J$ data. The $L$ curve shows the same effects as the $J$ one, but with a much reduced amplitude.

Figure 5 shows visual magnitude estimates for $\mathrm{R}$ For made by Danie Overbeek, an experienced South African variable star observer, over the same time period as the infrared data. His observations are among those reported by the AAVSO. Figure 6 shows these data after removing the 

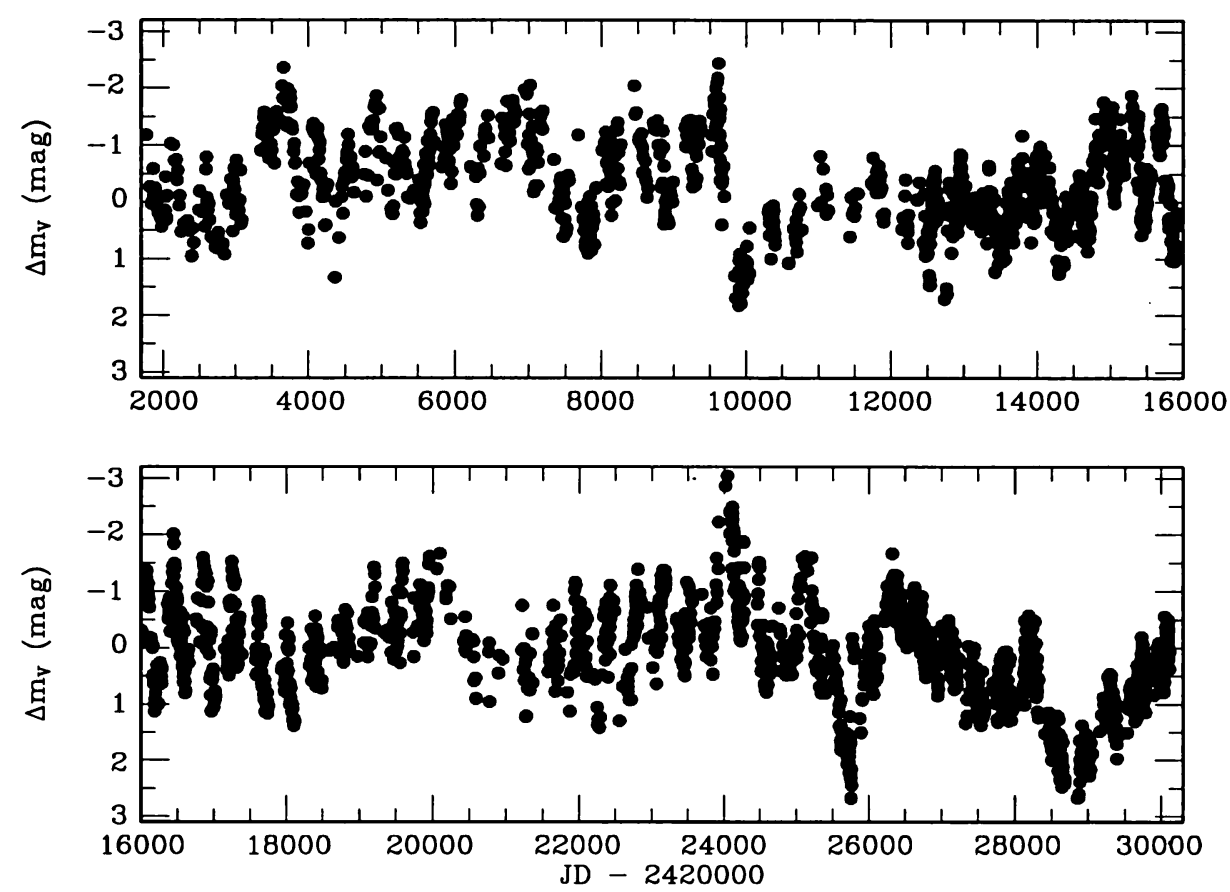

Figure 6. 76 years of AAVSO data for R For after removing the pulsational variations.

pulsations with a period of $386.6 \mathrm{~d}$ (no attempt was made to use different periods at different times). Although there is a lot of scatter in this diagram, as expected for visual magnitude estimates, it shows the same form as the $J$ light curve.

Feast et al. (1984) discussed the 1983 deep minimum in the visual and infrared light curves. They concluded that the most probable explanation was an increase in the obscuration of the star by a dust cloud containing predominantly large dust grains (diameters of the order of $0.15 \mu \mathrm{m}$ ). Presumably the more recent broad minimum has a similar explanation.

Winters et al. (1994) have suggested that discrete structures in the circumstellar dust shells may be produced by the formation of new dust which will decisively influence the shapes of the light curves by producing regular deep minima, as is shown in the theoretical light curves for different models illustrated in Figs. 5, 6, and 7 of their paper.

The light curves of $\mathrm{R}$ For, after removing the pulsational modulation (Figs. 3, 4, and 5), bear a striking similarity to those of $\mathrm{R} \mathrm{CrB}$ stars (e.g. Feast 1979). Indeed it seems possible that the explanation is the same, i.e. the star emits puffs of carbon-rich dust in random directions, and a minimum occurs when one of the puffs is emitted in our line of sight to the 
star. If the $\mathrm{R} \mathrm{CrB}$ explanation is correct, then we would expect the timing of the events to be random rather than periodic. There is certainly no sign of periodicities in the illustrated data of 20 years ( $\sim 19$ pulsation cycles).

The AAVSO database for $R$ For covers about 76 years and thus provides a much longer time base to examine for periodicities. Figure 6 shows the AAVSO data after the pulsational periodicity has been removed in the same way as it was for the infrared data. There is no sign of periodicity in the occurrences of faint phases even on this longer time base.

Other carbon Miras, in particular R Vol and R Lep, show similar behaviour to $\mathrm{R}$ For.

\section{TT Centauri}

We have fewer observations for the Mira TT Cen, but there is enough to suggest a rather different kind of behaviour from that of $\mathrm{R}$ For. The GCVS period is 462 days. There is no clear long-term trend at $J$ and a Fourier analysis finds 3 significant periods: $448.6,222.1$, and $149.5 \mathrm{~d}$. The light curve is shown in Figure 7, phased for a period of $448.6 \mathrm{~d}$. The fitted curve assumes this period and also makes use of the first three harmonics. The result is a secondary maximum on the rising branch of the curve. The $L$ data, perhaps surprisingly, do show some sign of fading of the mean light level. Most Miras show $L$ variations that are about half the amplitude of those at $J$. TT Cen is unusual in having comparable amplitudes at $J$ and $L$.

The $J$ light curve of TT Cen bears some resemblance to the theoretical curve produced by Wood (1995; see his Fig. 2) to illustrate the behaviour of the bolometric light curve of a model of an LMC Mira.

RV Cen (not illustrated) shows the same kind of behaviour both at $J$ and visually, but with more of a stand-still than a secondary peak.

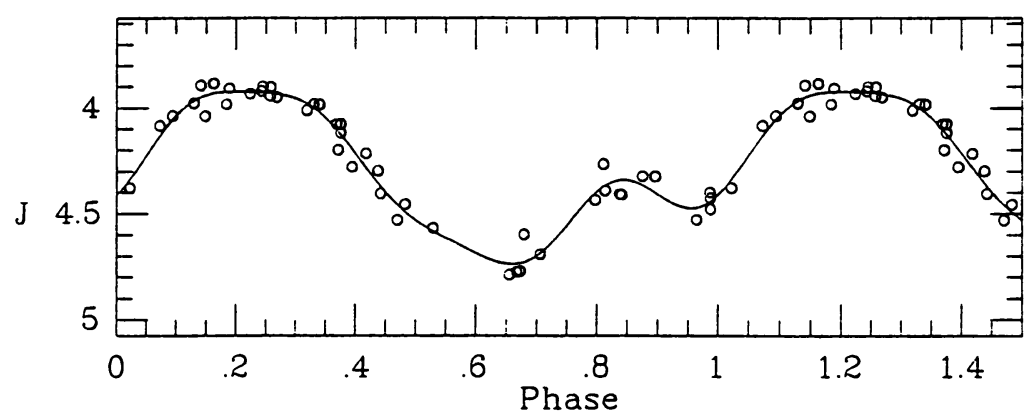

Figure 7. The $J$ light curve for TT Cen assuming a period of 448.6 days. 
Secondary maxima and stand-stills are common features of the light curves of Miras and many other types of variable star. Such features can be produced by a variety of mechanisms, such as shock-waves or periodic dust formation (e.g. Winters et al. 1994 and references therein) and are not in themselves of great diagnostic value.

\section{IZ Pegasi}

IZ Peg (CRL 3099) is a thick-shelled carbon Mira with very red colours. Whitelock et al. (1994) published a light curve and suggested it had two periods, 488 and $345 \mathrm{~d}$. Recent data do not support the second period, but a reasonable fit can be obtained with a 486-day pulsation and a long-term trend (Figure 8). A longer time base is required to determine the nature of the long-term trend.

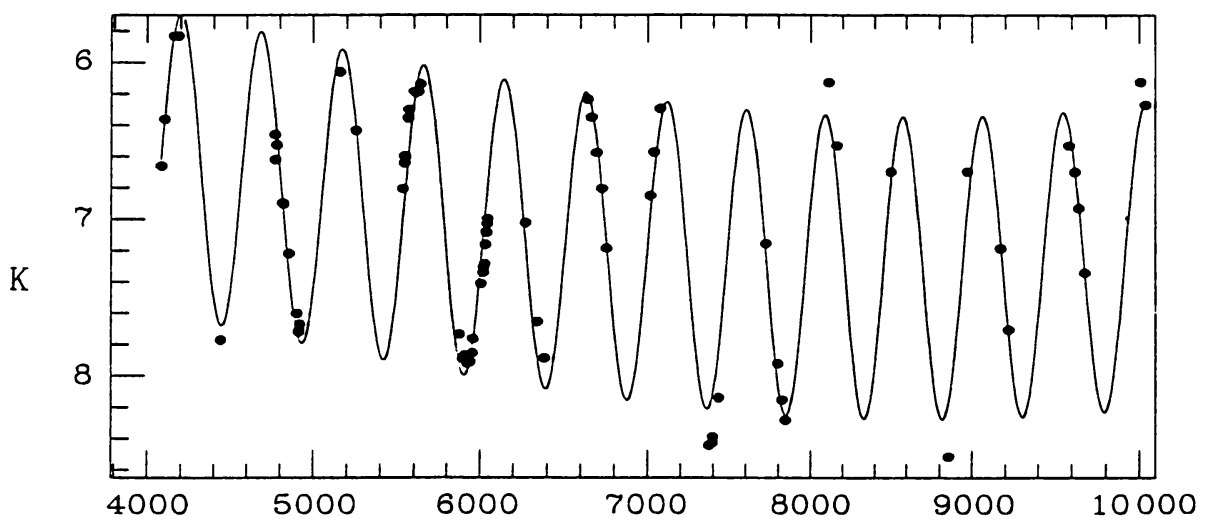

Figure 8. The $K$ light curve for IZ Peg; the abscissa is Julian Date minus 2440000. The fitted curve shows the 486-day pulsation and a long-term trend.

\section{R Sculptoris}

$\mathrm{R} \mathrm{Scl}$ is a well-known bright semi-regular variable. There has been an upsurge of interest in this star since the IRAS survey showed it to have unusual colours and various studies suggested that it was associated with hot and cool dust (e.g. Young et al. 1993). Essentially it has a detached dust shell (confirmed by Olofsson et al. 1996, who mapped the circumstellar CO) and therefore must have had a higher mass-loss rate in the not too distant past than it does at present. If we assume that it was a Mira in its previous high-mass-loss phase, then it seems possible that it may now be in a luminosity minimum following a helium shell flash. Alternatively it has left the Mira instability strip prior to its evolution across the HR diagram to 


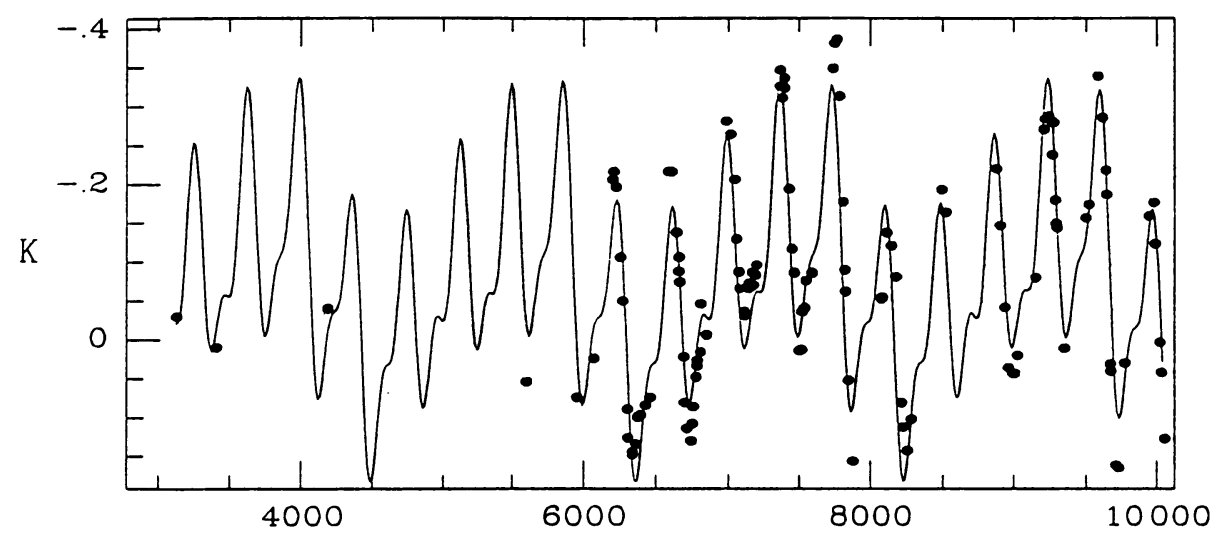

Figure 9. The $K$ light curve for $\mathrm{R}$ Scl; the abscissa is Julian Date minus 2440000 . The fitted curve shows the 374-day pulsations and an additional 1850-day modulation.

become a white dwarf. In any case it is likely to show interesting changes on a relatively rapid time scale. Figure 9 shows a $K$ light curve comprising Le Bertre's (1992) 28 observations and 93 measurements from SAAO (Whitelock et al. 1995, and unpublished).

The curve in Fig. 9 is derived from a 374.3 day pulsation and its first harmonic together with 1850-day variations. There are not enough data here to be sure that the 1850-day changes are really periodic. It is clear, however, that there is something other than the 374-day variations going on. Note that $\mathrm{R} \mathrm{Scl}$ is a small-amplitude variable, with a range less than $0.4 \mathrm{mag}$ at $K$ which is lower than that of any of the other stars discussed above. This curve has some similarities to the model illustrated in Fig. 7 of Winters et al. (1994).

\section{GM Canis Majoris}

Finally, consider another semiregular, GM CMa, otherwise known as IRC -20101. Unlike the stars described above, this is not a well-studied variable and no period has been published for it. I started observing it because its IRAS colours were very similar to those of $\mathrm{R} \mathrm{Scl}$. A Fourier analysis shows three significant periods, in order of decreasing amplitude, at 405, 780 and 201 days, and a marginal one at 100 days. The fit shown in Figure 10 is actually for 800 days and the three harmonics at 400, 200 and 100 days. Interestingly in this star the strongest peak is the first harmonic at 400 days which has three times the amplitude of the 800-day variations. This light curve bears some resemblance to those of the RV Tauri stars. Wood (1995) has found that oscillations at twice the primary period sometimes 


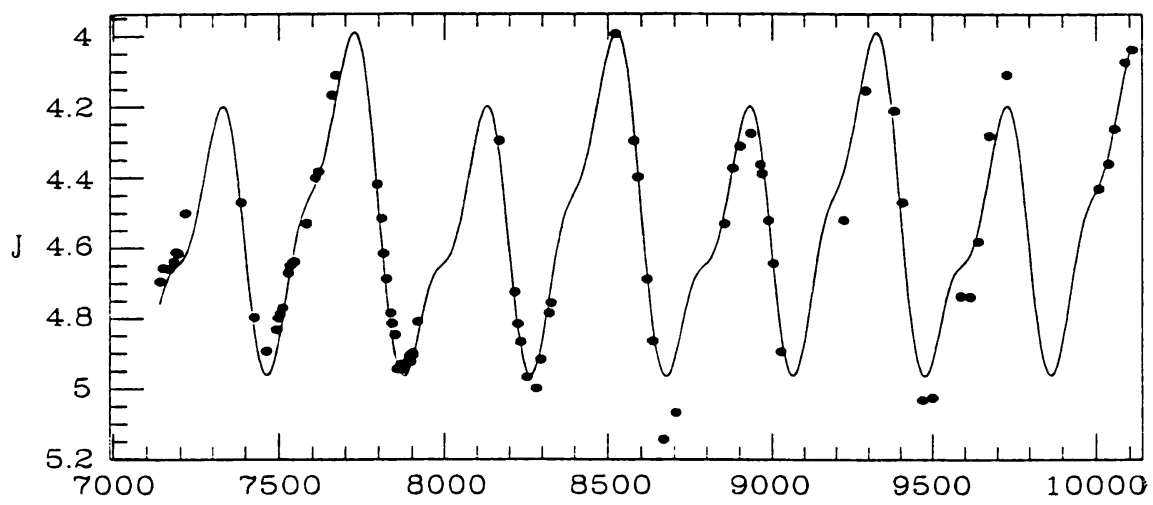

Figure 10. The $J$ light curve for GMI CMa; the abscissa is Julian Date minus 2440000. The fitted curve has periodicities of $800,400,300,200$ and 100 days.

occur in the outer layers of his stellar models. This may explain the 800-day variations found here if 400 days is the stellar pulsation period.

\section{Conclusions}

The infrared light curves of some C-rich variables show striking erratic variations associated with dust obscuration. There is as yet no evidence that these changes are periodic, and they might indicate that $\mathrm{R} \mathrm{CrB}$-like phenomena occur in carbon Miras. Other variables show double periods and peculiarities such as stand-stills in their light curves which are predicted by a variety of models. There are as yet insufficient data to be clear what is normal behaviour near the top of the AGB and what requires a more exotic explanation such as binary interaction.

I am very grateful to Danie Overbeek and to my colleagues at SAAO for the use of data prior to publication. I would also like to thank Janet Mattei and the AAVSO for allowing me to use their visual magnitudes for various carbon stars. I am grateful to Michael Feast and John Menzies for helpful discussions.

\section{References}

Feast, M. W. 1979, in IAU Coll. 46: Changing Trends in Variable Star Research, ed. F. M. Bateson, J. Smak, and I. H. Urch, Univ. Waikato (Hamilton, New Zealand), p. 246

Feast, M.W., Glass, I. S., Whitelock, P.A. \& Catchpole, R. M. 1989, Mon. Not. Roy. Astron. Soc., 241, 375

Feast, M. W., Whitelock, P. A., Catchpole, R. M., Roberts, G. \& Overbeek, M. D. 1984, Mon. Not. Roy. Astron. Soc., 211, 331

Groenewegen, M. A. T. \& Whitelock, P. A. 1996, Mon. Not. Roy. Astron. Soc., 281, 1347

Le Bertre, T. 1988, A.\& A., 190, 79 
Le Bertre, T. 1992, A\&A Supp., 94, 377

Olofsson, H., Bergman, P., Eriksson, K. \& Gustafsson, B. 1996, $A \& A, 311,587$

Whitelock, P., Menzies, J., Feast, M., Marang, F., Carter, B., Roberts, G., Catchpole, R. \& Chapman, J. 1994, MNRAS, 267, 711

Whitelock, P., Menzies, J., Feast, M., Catchpole, R., Marang, F. \& Carter, B. 1995, MNRAS, 276, 219

Whitelock, P. A. 1995, in IAU Coll. 155: Astrophysical Applications of Stellar Pulsation, ed. R. S. Stobie and P. A. Whitelock, ASP Conf. Ser., 83, 165

Winters, J. M., Fleischer, A. J., Gauger, A. \& Sedlmayr, E. 1994, A\&A, 290, 623

Wood, P. R. 1995, in IAU Coll. 155: Astrophysical Applications of Stellar Pulsation, ed. R. S. Stobie and P. A. Whitelock, ASP Conf. Ser., 83, 127

Young, K., Phillips, T. G. \& Knapp, G. R. 1993, ApJ, 409, 725

\section{Discussion}

Sedlmayr: Your material and the theoretical interpretations given are extremely valuable and very much needed in the present state of research. The calculations you mentioned by Winters et al. originally were not intended to model the specific light curves of real astronomical objects (like $\mathrm{R}$ For), but were aimed at providing a sufficient theoretical description of the coupled nonlinear processes in the shells of Miras and LPVs, which turned out to result in theoretical light curves exhibiting similar sub-variations. Also, your idea to correlate the irregular variations with a kind of $\mathrm{R} \mathrm{CrB}$ phenomenon seems very appealing but might raise its own theoretical problems in detail.

Whitelock: I think that the paper by Winters et al., and the series of which it is part, represent an important step forward in the understanding of these stars. I specifically mentioned the Winters et al. paper because it does discuss R For and the IR observations. I think that the extensive data we now have for this particular star suggest very strongly that periodic dust formation is not the correct explanation although it may be correct for other stars. 


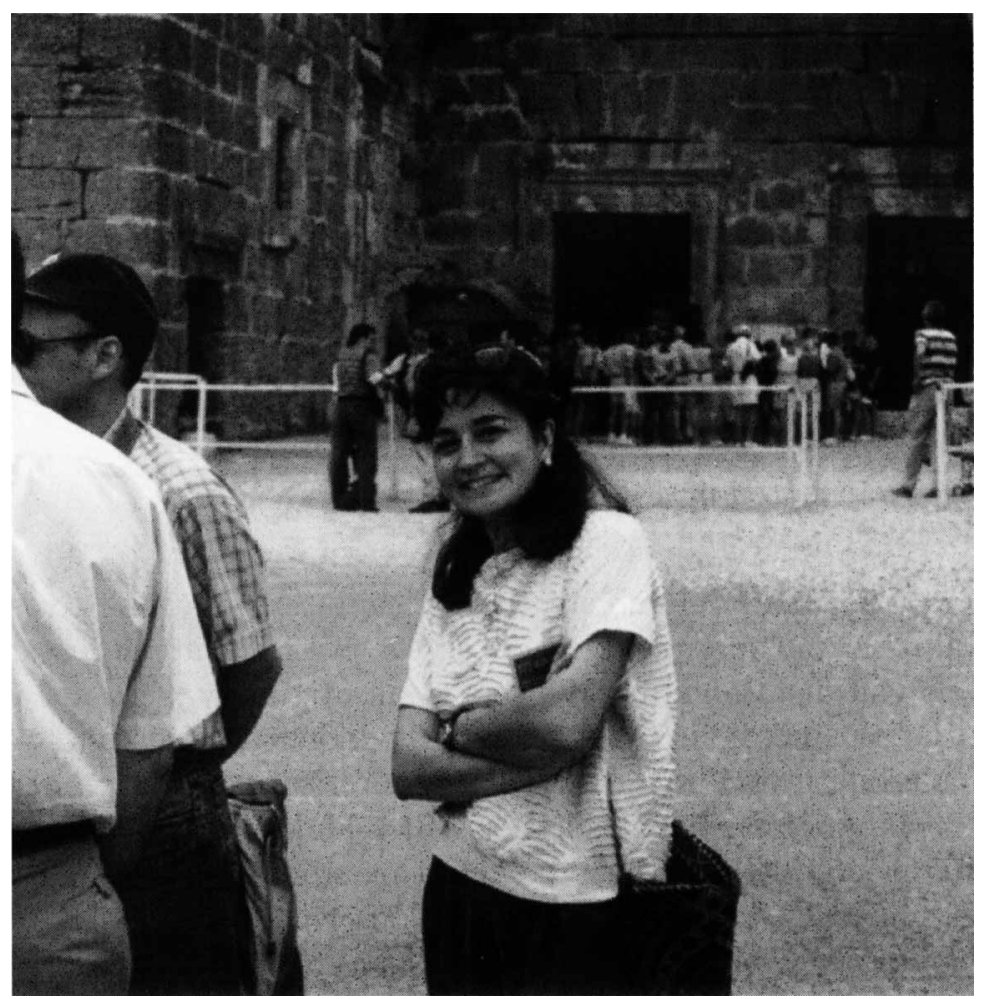

Hard-working Hülya Çalışkan outside the theater at Aspendos. Hülya's behind-the-scenes work on the LOC saved the symposium from possible disaster on several occasions. 